\title{
The juxtamembrane region of synaptotagmin 1 interacts with dynamin 1 and regulates vesicle fission during compensatory endocytosis in endocrine cells
}

\author{
Robyn L. McAdam ${ }^{1}$, Kelly T. Varga ${ }^{2}$, Zhongjiao Jiang ${ }^{2}$, Fiona B. Young ${ }^{1}$, Vanessa Blandford ${ }^{1}$, \\ Peter S. McPherson ${ }^{1}$, Liang-Wei Gong ${ }^{2}$ and Wayne S. Sossin ${ }^{1, *}$
}

\begin{abstract}
Synaptotagmin 1 (Syt1) is a synaptic vesicle protein that is important for the kinetics of both exocytosis and endocytosis, and is thus a candidate molecule to link these two processes. Although the tandem $\mathrm{Ca}^{2+}$-binding $\mathrm{C} 2$ domains of Syt1 have important roles in exocytosis and endocytosis, the function of the conserved juxtamembrane (jxm) linker region has yet to be determined. We now demonstrate that the jxm region of Syt1 interacts directly with the pleckstrin homology $(\mathrm{PH})$ domain of the endocytic protein dynamin 1. By using cell-attached capacitance recordings with millisecond time resolution to monitor clathrin-mediated endocytosis of single vesicles in neuroendocrine chromaffin cells, we find that loss of this interaction prolongs the lifetime of the fission pore leading to defects in the dynamics of vesicle fission. These results indicate a previously undescribed interaction between two major regulatory proteins in the secretory vesicle cycle and that this interaction regulates endocytosis.
\end{abstract}

KEY WORDS: Compensatory endocytosis, Chromaffin cells, Synaptotagmin, Syt1, Dynamin

\section{INTRODUCTION}

Exocytosis and compensatory endocytosis of neurotransmittercontaining vesicles are highly regulated processes in neurons and neuroendocrine cells. Endocytosis is linked with exocytosis such that a proportionate amount of endocytosis occurs in response to exocytosis (Gundelfinger et al., 2003; Barg and Machado, 2008; Smith et al., 2008; Cardenas and Marengo, 2010). Although the mechanisms for linking endocytosis to exocytosis are largely unknown, there has been considerable interest in the role of synaptotagmins (Syts) in this process, and in particular for Syt1 because of its crucial role in both exocytosis and endocytosis (Poskanzer et al., 2003; Nicholson-Tomishima and Ryan, 2004; Yao et al., 2012a). More specifically, the Syt1 C2 domains have been implicated in endocytosis through binding to endocytic adaptor proteins stonin 2 (Diril et al., 2006; Jung et al., 2007) and AP-2 (Zhang et al., 1994). Recently, it was found that the $\mathrm{Ca}^{2+}$ dependence of endocytosis can be attributed to Syt1 (Yao et al., 2012a,b).

Between the transmembrane domain and the tandem $\mathrm{C} 2$ domains, Syt1 has a juxtamembrane (jxm) linker. Evolutionarily, the Syt 1 jxm linker is conserved, indicating that this region might play

${ }^{1}$ Department of Neurology and Neurosurgery, Montreal Neurological Institute, McGill University, Montreal, Quebec, H3A 2B4, Canada. ${ }^{2}$ Department of Biological Sciences, University of Illinois at Chicago, Chicago, IL 60607, USA.

*Author for correspondence (wayne.sossin@mcgill.ca)

Received 11 August 2014; Accepted 30 April 2015 important roles. Three conserved features are a PKC and CaMKII phosphorylation site (Hilfiker et al., 1999), an asymmetric distribution of charges leading to an electrostatic zipper (Lai et al., 2013), and a splice site where wobble-splicing with alternative exon entry can lead to the variable inclusion of two or three additional amino acids - VQ in invertebrates (Nakhost et al., 2003) and ALK in vertebrates (Lv et al., 2009). In vertebrates, Syt1 is a member of a three-protein family composed of Syt1, Syt2 and Syt9, which has arisen because of a gene triplication in the chordate lineage from a single family member in invertebrates. These Syt1 family members differ in their jxm region: Syt9 retains the splice insert 'VQ' seen in invertebrates, but is no longer a target of wobble splicing; Syt2 has a deletion in this region removing both the phosphorylation site and the wobble splice site; and Syt1 conserves wobble splicing leading to two splice variants, with or without an 'ALK' insert (Fig. 1A). To further shed light on the function of the Syt1jxm region, we have searched for binding partners of the region in vertebrates. We show that the Syt 1 jxm region specifically binds to the pleckstrin homology $(\mathrm{PH})$ domain of dynamin 1 , and that binding is regulated by the phosphorylation site, but not by the wobble splicing. Functionally, we show that this interaction regulates clathrin-mediated endocytosis (CME) of chromaffin granules by influencing fission pore dynamics.

\section{RESULTS AND DISCUSSION}

\section{The Syt1jxm region interacts in vitro with dynamin 1}

To determine the function of the conserved Sytljxm region (Fig. 1A), we sought to identify binding partners for this domain using affinity-selection assays from rat brain homogenates with GST fusion proteins of the jxm region of rat Syt1, with or without the ALK insert. SDS-PAGE gels were stained with Coomassie Blue and bands that appeared in the GST-Syt1jxm and GST-Syt1jxmALK lane, but not in the GST control lane, were excised and submitted to tandem mass spectrometry analysis (Fig. 1B). Both the band isolated from the GST-Syt1jxm and GST-Syt1jxm-ALK lane contained peptides from the major endocytic protein dynamin 1, whereas no dynamin 1 peptides were identified in the GST-Syt 2 or the GST alone lane. Over 30 unique peptides covering $50 \%$ of the endocytic protein dynamin 1 were identified (Fig. 1C). The slightly different molecular mass of the dynamin 1 bands in the GSTSyt1jxm-ALK versus the GST-Syt1jxm lane are due to distortions caused by the large amount of GST fusion protein loaded in these lanes. Confirming this result, an antibody to Syt1 was able to immunoprecipitate dynamin 1 (Fig. 1D). To investigate species and isoform specificity, GST fusion constructs were generated using the jxm region of invertebrate Aplysia Syt1, with and without the wobble splice, as well as rat Syt4. Pulldown assays in rat brain homogenate show that none of these constructs bind mammalian 

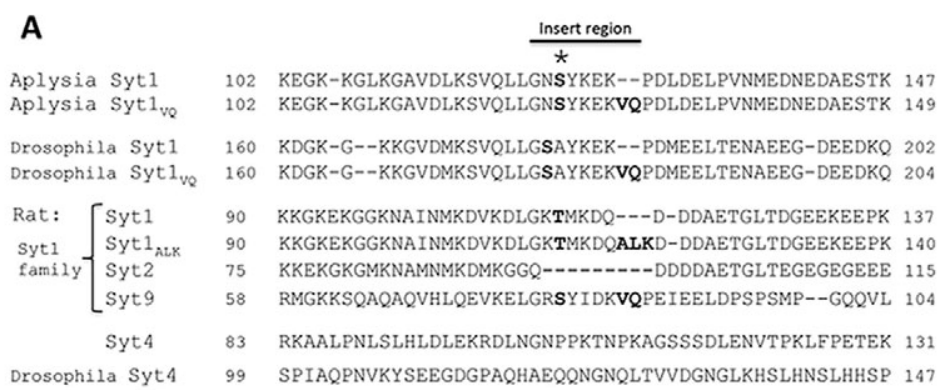

B

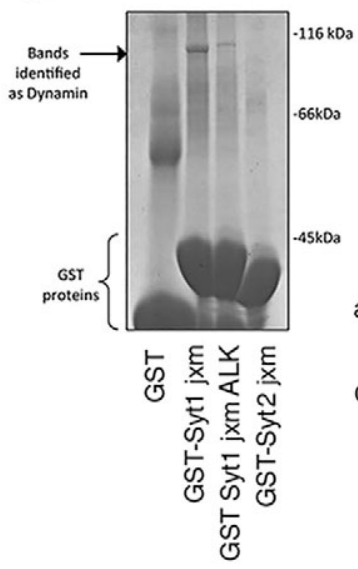

D

anti-dynamin

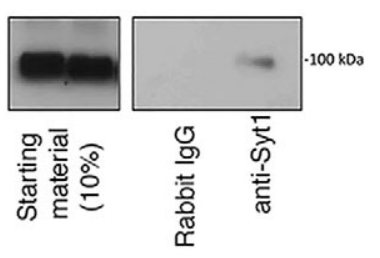

E

anti-dynamin

Ponceau:

GST proteins
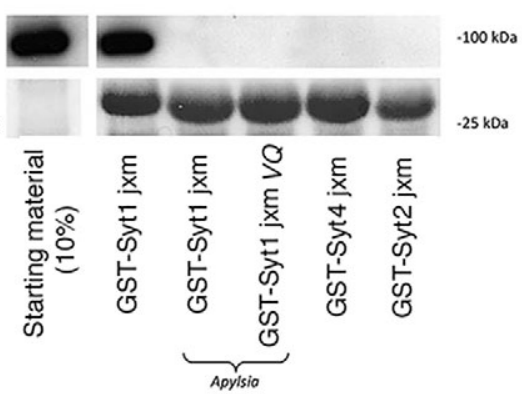

Fig. 1. The Syt1 juxtamembrane domain is conserved and interacts in vitro with dynamin. (A) Alignment of amino acid sequence from various species highlighting conservation and splice variation. The $\left(^{*}\right)$ indicates the phosphorylation site.

(B) Rat brain extracts were incubated with GST-Sytjxm constructs and bound proteins were separated on SDS-PAGE gels and stained with Coomassie Blue and shows bands excised for tandem mass spectrometry analysis and identified as dynamin. (C) The amino acid sequence of rat dynamin 1 is shown with peptides obtained by mass spectrometry highlighted. (D) Synaptosomes were used for immunoprecipitation using anti-Syt1 antibody or IgG control followed by immunoblotting with anti-dynamin to confirm the Syt1-dynamin-1 interaction.

(E) Pulldown experiments from rat brain extract using purified GST fusion proteins of the jxm from multiple Syt isoforms and immunoblotted with anti-dynamin antibody. The loading of the GST fusion proteins is demonstrated with a Ponceau stain of the membrane.

\section{C}

MGNRGMEDLIPLVNRLQDAFSAIGQNADLDLPQIAVVGGQSAGKSSVLENFVGRDFLPRGSGIVTRRPLV LQLVNSTTEYAEFLHCKGKKFTDFEEVRLEIEAETDRVTGTNKGISPVPINLRVYSPHVLNLTLVDLPGM TKVPVGDQPPDIEFQIRDMLMQFVTKENCLILAVSPANSDLANSDALKIAKEVDPQGQRTIGVITKLDLM DEGTDARDVLENKLLPLRRGY I GVVNRSQKDIDGKKDITAALAAERKFFLSHPSYRHLADRMGTPYLOKV LNOOLTNHIRDTLPGLRNKLOSOLLSIEKEVDEYKNFRPDDPARKTKALLOMVOOFAVDFEKRIEGSGDQ I IDTYELSGGARINRIFHERFPFELVKMEFDEKELRREISYAIKNIHGIRTGLFTPDLAFEATVKKQVQKL KEPSIKCVDMVVSELTSTIRKCSEKLQQY PRLREEMERIVTTHIREREGRTKEQVMLLIDIELAYMNTNH EDFIGFANAQQRSNQMNKKKTSGNQDEILVIRKGWLTINNIGIMKGGSKEYWFVLTAENLSWYKDDEEKE KKYMLSVDNLKLRDVEKGFMSSKHIFALFNTEQRNVYKDYRQLELACETQEEVDSWKASFLRAGVYPERV GDKEKASETEENGSDSFMHSMDPQLERQVETIRNLVDSYMAIVNKTVRDLMPKTIMHLMINNTKEFIFSE LLANLYSCGDQNTLMEESAEQAQRRDEMLRMYHALKEALSI IGDINTTTVSTPMPPPVDDSWLQVQSVPA GRRSPTSSPTPQRRAPAVPPARPGSRGPAPGPPPAGSALGGAPPVPSRPGASPDPFGPPPQVPSRPNRAP PGVPRITISDP

dynamin 1, indicating the species and isoform specificity of the dynamin 1 and Syt1 interaction (Fig. 1E).

The jxm regions of Syt1 and Syt2 are highly conserved, but only the jxm region of Syt1 binds dynamin 1. A major difference in the jxm region of Syt1 and Syt2 is that Syt2 has a nine-aminoacid deletion causing a loss of the wobble splicing and the conserved phosphorylation site (Fig. 1A). We therefore examined the importance of this region for the Sytljxm interaction with dynamin 1. Surprisingly, replacing the insert region of Syt1 with the shorter sequence in Syt2 did not disrupt the interaction (Fig. 2A). To determine whether changes in this region could regulate binding, we compared binding of Sytljxm with and without the wobble splice insert. There was no difference in dynamin 1 binding between Syt1jxm and Syt1jxm ALK (Fig. 2A), despite less dynamin 1 binding on the original pulldown (Fig. 1B). We also replaced the conserved threonine (T112) phosphorylation site for a phosphomimetic glutamic acid (E) or a non-phosphorylatable alanine (A). Strikingly, the phosphomimetic mutation, Syt1jxm $\mathrm{T} \rightarrow \mathrm{E}$, is unable to bind dynamin, whereas the Syt1jxm $\mathrm{T} \rightarrow \mathrm{A}$ mutant maintained binding
(Fig. 2A), suggesting that Syt1 phosphorylation regulates binding to dynamin 1 .

\section{Syt1jxm interacts directly with the dynamin 1 PH domain}

We next sought to determine which domain of dynamin 1 interacts with the Syt1jxm. Dynamin contains five functional domains: a GTPase domain, a middle domain (MID), a pleckstrin homology $(\mathrm{PH})$ domain, a GTPase effector domain (GED) and a proline-rich domain (PRD). To localize the binding region, we performed pulldown assays in which GST-Syt1jxm was incubated with lysates from HEK-293T cells expressing various flag-tagged dynamin mutant constructs with successive C-terminal truncations (Fig. 2B). We found that removal of the GED and PRD from dynamin did not affect its ability to bind GST-Syt1jxm, but removal of the GED, $\mathrm{PRD}$, and the PH domain produced a loss of interaction, suggesting that the Syt1jxm is interacting with dynamin 1 through its $\mathrm{PH}$ domain (Fig. 2B). However, when just the $\mathrm{PH}$ domain is removed, binding is even stronger (Fig. 2C), suggesting that additional regions that mediate the interaction with dynamin 1 are present within the Syt1jxm domain and are affected by removal of the $\mathrm{PH}$ 

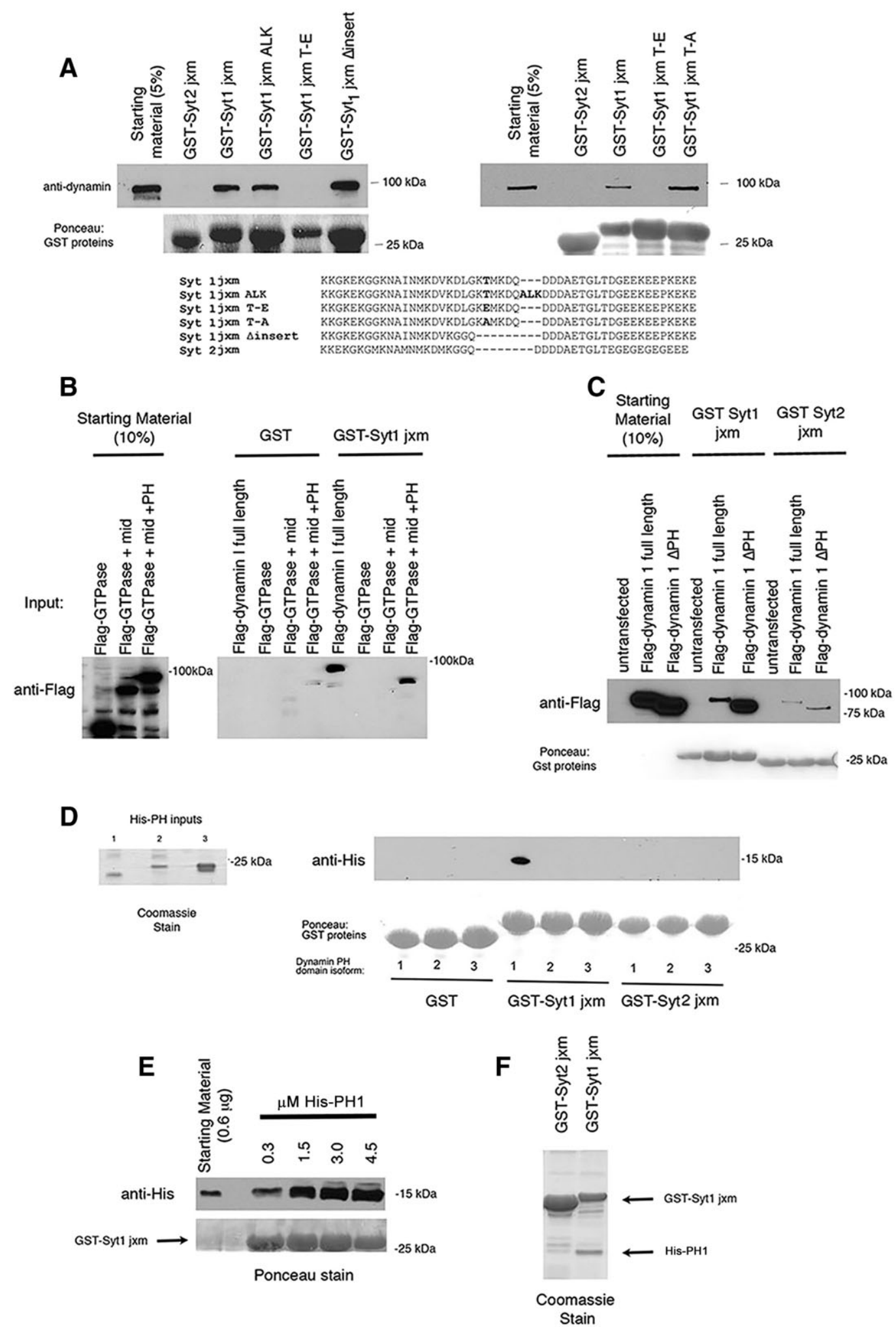

Fig. 2. Characterization of the Syt1jxm-dynamin-1 interaction. (A) Pulldown experiments from rat brain extract using purified GST fusion proteins followed by immunoblotting with anti-dynamin. Loading of GST fusion proteins is shown by Ponceau staining below. Jxm sequences of constructs are shown. (B) Pulldown experiments from HEK-293T cells transiently transfected with various dynamin constructs (defined in Materials and Methods) using purified GST fusion proteins followed by immunoblotting with anti-dynamin antibody. Starting material is shown on the left. (C) Pulldown experiments from HEK-293T cells transiently transfected with various dynamin constructs (defined in Materials and Methods) using purified GST fusion proteins followed by immunoblotting with anti-dynamin. Ponceau staining shows loading of GST fusion proteins. (D) Pulldown assay with purified GST fusion proteins and purified His-tagged proteins of the dynamin 1,2 and $3 \mathrm{PH}$ domains as input followed by immunoblotting with anti-His antibody. Coomassie stain of the input is shown to the right. (E) Increasing amounts of the His-PH1 domain were added to a constant amount of GST-Syt1jxm domain for pulldown assays. Ponceau stain is shown for loading of GST fusion proteins. (F) A Coomassie stained gel after pulldown of GST-Syt1jxm or GST-Syt2jxm with a saturating amount of His-PH1 (5 $\mu$ M) shows stoichiometry of the interaction. 
A

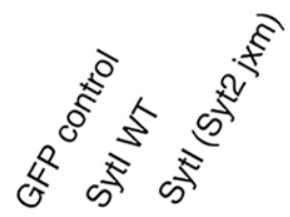
Anti-myc

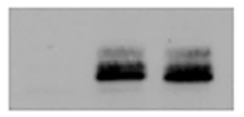

$67 \mathrm{kDa}$
B

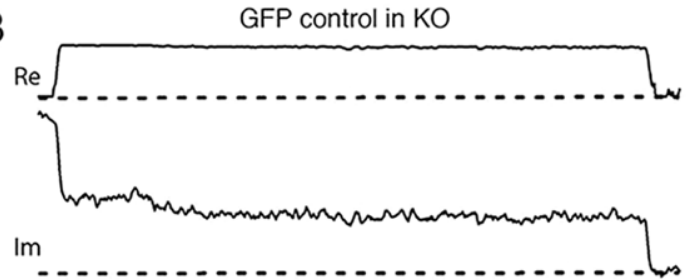

Gp

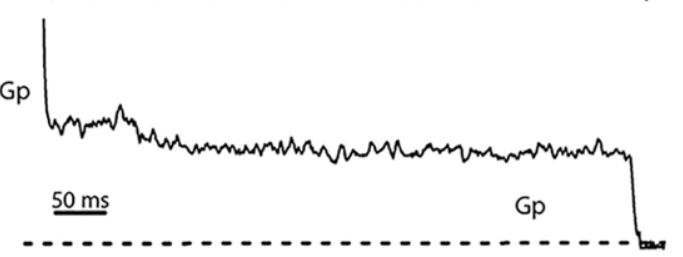

Sytl (Syt2 jxm) in KO
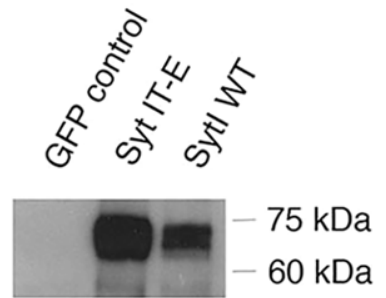

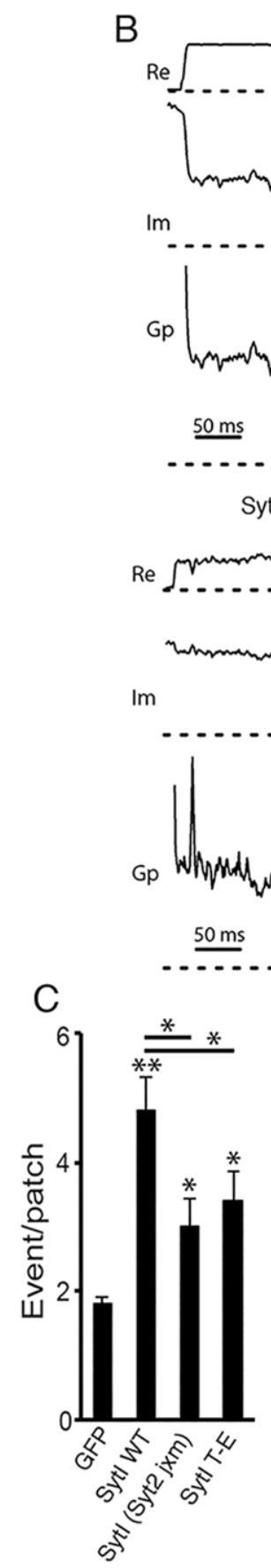

Sytl WT in KO

| $30 \mathrm{pS}$

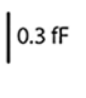

$80 \mathrm{pS}$
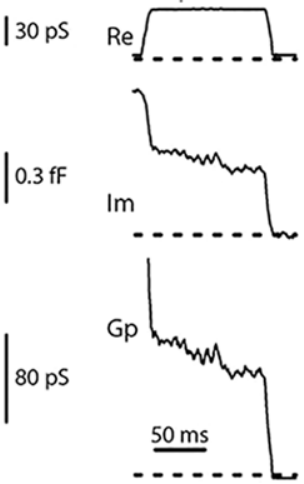

Sytl T-E in KO
Re
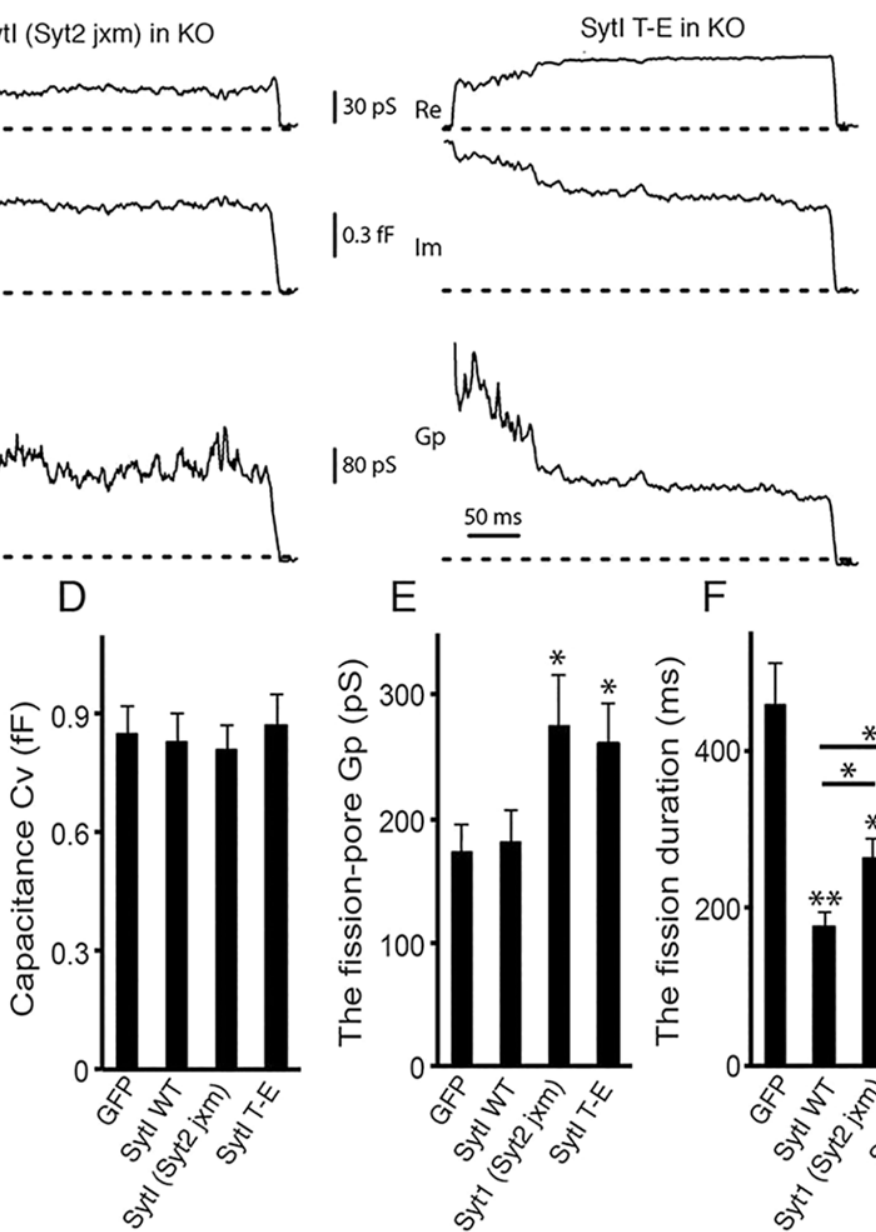

|80ps

E

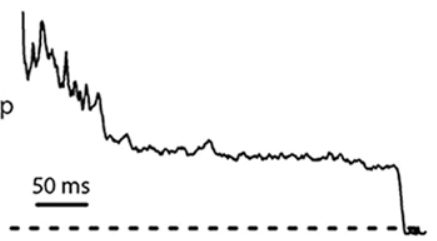

E

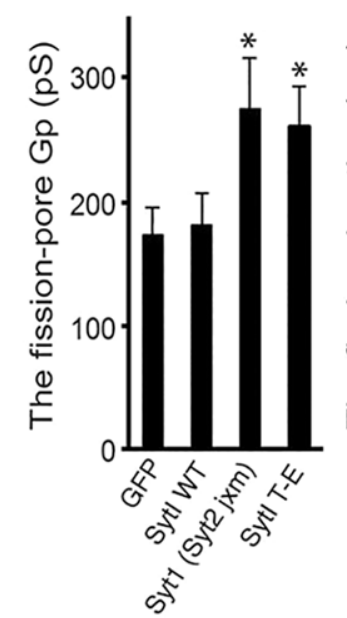

$\mathrm{F}$

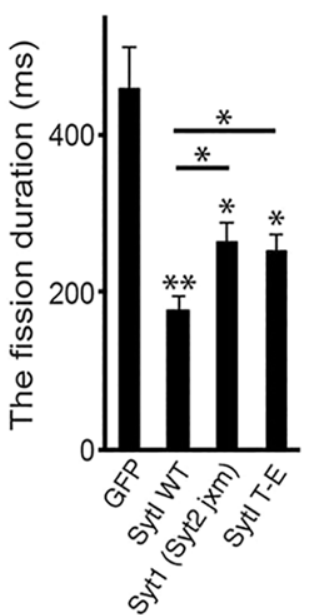

Fig. 3. Fission-pore duration and conductance are increased in interactiondeficient cells. (A) Lysates obtained from transduced hippocampal cultures were immunoblotted with anti-Myc antibody to demonstrate expression levels of Syt1WT and Syt1 mutants. (B) Representative fission pore events in Syt1 KO (top left), rescue (WT Syt1 in Syt1 KO, top right) and interaction deficient [Syt1(Syt2jxm) in KO (bottom left) and Syt1T$\mathrm{E}$ (bottom right)] cells recorded in a cellattached patch configuration. The traces from top to bottom show the time course of: $\operatorname{Re}$ (real part of membrane current), Im (imaginary part of membrane current), and Gp (fission-pore conductance). For detailed descriptions, see Yao et al., 2012b, 2013. (C-F) Summary of results (mean \pm s.e.m.). ${ }^{*} P<0.05,{ }^{*} P<0.01$

(Tukey's post hoc test after one-way ANOVA, $n>38$ ). Comparisons to GFP alone (Syt1KO cells) are shown directly over the histogram and comparisons between the rescue constructs are shown over the lines. domain. To determine whether there is a direct interaction between Sytljxm and the PH domain, we bacterially expressed and purified His-tagged dynamin 1 PH domain construct (His-PH1) and found that it interacted with GST-Sytljxm (Fig. 2D). Given that this interaction occurs between two proteins both purified from bacteria, it indicates that the interaction is direct and does not require any 
additional neuronal proteins. Like full-length dynamin 1, His-PH1 does not interact with GST-Syt2jxm (Fig. 2D). There is considerable homology between the PH domains of the three dynamin isoforms, yet we observed that the PH domain of dynamin 1, but not of dynamin 2 or 3, interacts with Syt1jxm (Fig. 2D). The interaction between the Syt1jxm domain and the PH domain saturates at about $5 \mu \mathrm{M}$ concentration of His-PH1 (Fig. 2E) and at this concentration the stoichiometry is $25+8 \%(n=3$, \pm s.e.m.) HisPH1 relative to Syt1jxm (Fig. 2F).

The interaction of the Syt1jxm domain with either dynamin or its $\mathrm{PH}$ domain is only seen at low concentrations of salt $(<50 \mathrm{mM})$. Importantly, a number of physiological interactions, particular in the regulation of synaptic vesicle exocytosis and endocytosis are known to be sensitive to salt [for example, synapsin binding to synaptic vesicles (Huttner et al., 1983) is completely disrupted by $50 \mathrm{mM} \mathrm{NaCl}$, and the ability of AP-2 to bind to NECAP1 or clathrin, both undoubtedly important physiological interactions, are sensitive to salt in the 30-50 mM range (Ritter et al., 2013)].

\section{Blocking the interaction of Syt1 with dynamin 1 affects CME fission pore dynamics in neuroendocrine cells}

After establishing in vitro that Syt1jxm interacts directly and specifically with dynamin 1 , we investigated the functional relevance of the interaction. Dynamin-ring formation and subsequent GTPase-mediated constriction around the neck of a budding vesicle during $\mathrm{CME}$ enables fission pore closure and membrane scission. The $\mathrm{PH}$ domain localizes dynamin to the plasma membrane by binding phospholipids, in turn stimulating GTPase activity and fission-pore closure (Ramachandran et al., 2009). Interestingly, most neuroendocrine cells contain Syt1 but not Syt2 (Marqueze et al., 1995) suggesting that the specific interaction between Syt1 and dynamin 1 might be because there is a specific role for the Syt1-dynamin-1 interaction in regulating endocytosis in neuroendocrine cells. Moreover, Syt1 is implicated in regulating fission-pore dynamics in these cells as loss of Syt1 in neuroendocrine chromaffin cells results in a significant increase in the fission-pore duration during CME (Yao et al., 2012b). Given that both Syt1 and dynamin 1 are involved in the fission process of CME, we hypothesized that the Syt-dynamin interaction might have a role in mediating fission dynamics. To test this, we performed electrophysiological capacitance recordings in chromaffin cells to measure kinetics of single vesicle retrieval while the interaction was inhibited. Chromaffin cells from Syt1knockout $(\mathrm{KO})$ mice were transduced with a lentivirus encoding either a wild-type human Syt1 (rescue condition) or two mutant Syt1s with jxm domains that do not bind dynamin, either with the entire Syt 2 jxm domain replacing the Syt1 domain [Syt1(Syt 2 jxm)] or the Syt1jxm domain with a $\mathrm{T} \rightarrow \mathrm{E}$ mutation at the putative phosphorylation site (Syt1T-E), all with an N-terminal Myc tag. The lentivirus also expressed a reporter GFP under an IRES promoter. All these proteins express at approximately equal levels, although there is slightly higher expression of the Syt1T-E mutant (Fig. 3A). The number of endocytic events is decreased in Syt1 KO cells and rescue with human Syt1 restores the number of events to WT levels (Yao et al., 2012b). The two non-interacting mutants had fewer endocytic events than the WT although more than the Syt1 $\mathrm{KO}$, suggesting a partial dependence on Syt1jxm-dynamin-1 binding for the number of endocytic events (Fig. 3C). There was no difference in the capacitance size of endocytic vesicles between all these four groups (Fig. 3B,D). Thus, the capacitance size of endocytic vesicles is not altered, indicating the Syt1-dynamin-1 interaction might not be important for the size or diameter of endocytic vesicles. Unlike Syt1 KO cells and Syt1 KO cells expressing wild-type Syt1 (Syt1WT), cells expressing either mutant Syt1 showed an increase in fission pore conductance, indicative of a wider pore stalk (Fig. 3B,E). The prolonged fission pore duration seen in Syt1 KO cells (Yao et al., 2012b) was completely rescued by expression of human Syt1 whereas expression of the Syt1 mutants with defective dynamin binding had a significantly increased fission pore duration (Fig. 3B,F), although this was still significantly different than the KO. Taken together, these results suggest that the Syt1-dynamin-1 interaction facilitates vesicle fission during CME.

The effects we see are likely independent to be of any effects of the Syt1jxm domain on fusion as Syt 2 can replace Syt 1 for fusion with little effect in chromaffin cells, and indeed the phosphomimetic mutation that disrupts the Syt1-dynamin-1 interaction also does not affect fusion in these cells (Nagy et al., 2006). Moreover, there were no differences in exocytosis in Syt1 KO cells expressing Syt1WT or Syt1 mutants with defective dynamin binding as measured by carbon fiber amperometry [WT Syt1 $=33.1 \pm 6.0$ spikes, $n=25$; Syt1 $($ Syt 2 jxm $)=30.1 \pm 5.7$ spikes, $n=22 ;$ Syt1T-E $=32.8 \pm 4.2$ spikes, $n=22 ; P>0.05$ (mean \pm s.e.m.)]. Previous studies in chromaffin cells have suggested a specific dynamin-dependent clathrin-independent role of the PH domain of dynamin 1, but not dynamin 2, in mediating fission of dense core vesicles that have not completely fused with the plasma membrane, often called rapid endocytosis or 'kiss-and-run' endocytosis (Artalejo et al., 1997). However, the assay in our study measures a clathrin-dependent retrieval of chromaffin granule membranes (Yao et al., 2012b). In the future, it will be interesting to see if the Syt1-dynamin-1 interaction is important in the clathrinindependent endocytosis of chromaffin granules.

In conclusion, we have demonstrated a specific interaction between the jxm domain of Syt1 and dynamin 1 that is important for the Syt1dependent regulation of the chromaffin granule fission pore.

\section{MATERIALS AND METHODS}

\section{Expression constructs}

To generate N-terminal GST fusion constructs, the jxm region from IMAGE clones of mammalian Syt1, Syt1-ALK, Syt2 and Syt4 or Aplysia cDNA was amplified and ligated into vector pGEX-2T at restriction enzyme sites BamHI and EcoRI. Mutations were generated using overlap PCR and validated by sequencing (Fig. 2A).

The N-terminally Flag-tagged dynamin 1 (Genbank numberNP_542420.1) construct was generated by PCR amplification of the full-length dynamin or its truncations (Flag-GTPase+mid+PH:1-662, Flag-GTPase+mid:1-498, FlagGTPase:1-247 and Flag- $\Delta \mathrm{PH}: 1-516 ; 630-851)$ followed by ligation into the vector pCMV-Tag2C. N-terminally His-tagged $(6 \times$ His) dynamin $\mathrm{PH}$ domain constructs were generated by PCR using isoform-specific primers and rat brain cDNA (HisPH1:508-626; HisPH2:510-634; HisPH3:518-635) followed by ligation into $\mathrm{pTrcHisB}$ vector (Invitrogen).

To generate Syt1 vectors for lentivirus production, we generated a Myctagged human Syt1WT with PCR using a human Syt1 template (IMAGE clone 6187902; GI37589129) and a 5' primer encoding the Myc tag. This PCR product was ligated into the EMCV IRES-EGFP-containing plasmid pWPI (Addgene) and then subcloned into the lentiviral expression vector pRRLsinPPT. To generate the Syt1(Syt2 jxm) expression construct, overlap PCR was used to exchange the Syt1jxm for the Syt2 sequence. The jxm in the Syt1WT is: KKKNKKKGKEKGGKNAINMKDVKDLGKTMDQALKDDDAETGLTDGEEKEEPKEEE. The jxm in the Syt1(Syt2jxm) is: KKKKNKKKEKGKGMKNAMNKDMKGGQDDDDAETGLDGEEKEEPKEEE. Site-directed mutagenesis was used to generate the Syt1T-E mutant.

\section{GST pulldown experiments}

E. coli strain BL21 was used to express fusion proteins. GST proteins were purified using glutathione-Sepharose 4B beads (GE). His-tagged proteins 
were purified using nickel-affinity chromatography, and buffer exchange was performed with PD-10 columns (GE) into PBS. Incubations with beads were performed by rotating at $4^{\circ} \mathrm{C}$. Immediately prior to use in pulldown assays, beads were pre-blocked by incubating in $1 \mathrm{ml}$ of lysis buffer containing 1\% BSA for $30 \mathrm{~min}$ then washed in $1 \mathrm{ml}$ lysis buffer for $15 \mathrm{~min}$.

Rat brain extract was obtained by homogenization of whole rat brains (Pel-Freez; $\sim 2 \mathrm{~g}$ ) at $4^{\circ} \mathrm{C}$ in $20 \mathrm{ml}$ of lysis buffer $(20 \mathrm{mM}$ HEPES $\mathrm{pH} 7.4$, a protease inhibitor cocktail tablet, $0.1 \%$ PMSF, and $0.25 \%$ $\beta$-mercaptoethanol) and centrifugation at $205,000 \mathrm{~g}$ for $30 \mathrm{~min}$ at $4^{\circ} \mathrm{C}$. GST fusion proteins $(20-30 \mu \mathrm{g})$ were incubated with $500 \mu \mathrm{l}$ of rat brain extract and $500 \mu \mathrm{l}$ lysis buffer and rotated at $4{ }^{\circ} \mathrm{C}$ for $1 \mathrm{~h}$. After washing three times for $5 \mathrm{~min}$ in lysis buffer, protein was eluted from the beads by adding $10 \mu \mathrm{l}$ of $5 \times$ Laemmli Sample buffer and incubated at $95^{\circ} \mathrm{C}$ for $5 \mathrm{~min}$. For purified His-tagged proteins, the same protocol was used except that His protein was added to GST-Syt in $1 \mathrm{ml}$ of lysis buffer containing $10 \mathrm{mM} \mathrm{NaCl}$. For Flagtagged dynamin proteins, HEK-293T cells were transfected and left for 24-48 h. Cells were washed three times with PBS before suspension into $1 \mathrm{ml}$ of lysis buffer and sonication. The cell mixture was centrifuged at $205,000 \mathrm{~g}$ for $60 \mathrm{~min}$, and $100 \mu \mathrm{l}$ of the supernatant was added to GST-Syt protein in $900 \mu \mathrm{l}$ of lysis buffer and the same protocol as stated above was used. Eluted proteins were separated by SDS-PAGE and transferred onto nitrocellulose membrane. Mass spectrophotometry was performed as described previously (Elvira et al., 2006).

\section{Immunoprecipitation experiments}

Immunoprecipitations using anti-Syt1 (StressGen Biotechnologies, 1:200) from P2 fractions of rat brains was performed as described previously (de Heuvel et al., 1997).

\section{Immunoblotting}

The primary anti-bodies used were anti-dynamin (Upstate, 1:16,000), antiHis (Invitrogen, 1:1000), anti-Myc (Sigma, 1:1000), and anti-Flag (Sigma, 1:1000). Primary and HRP-conjugated secondary antibodies (1:5000 1:8000) were diluted in TBS with Tween-20 containing 5\% skimmed milk powder and incubated with nitrocellulose membrane for $1 \mathrm{~h}$ at room temperature. Western Lightning Plus-ECL kit (PerkinElmer) was used as per manufacturer's instructions to detect protein bands.

\section{Lentivirus experiments}

Lentiviruses were produced and titered as described (Ritter et al., 2013). For chromaffin cell transduction, several hours after plating, 3-5 viral particles per cell were applied to the cells for $3 \mathrm{~h}$ and patch-clamp measurements were performed 12-24 h later. 5-day-old primary rat hippocampal neuron cultures were transduced similarly and were lysed at 21 days in vitro for immunoblotting.

\section{Chromaffin cell preparation, cell-attached recordings, and fission-pore analysis}

Adrenal glands were utilized from newborn pups of either sex (post-natal day 0) of Syt1 heterozygous (HT) mouse matings and were isolated according to the guidelines of the National Institutes of Health, as approved by the Animal Care and Use Committee of the University of Illinois at Chicago. Mouse chromaffin cells were prepared and cultured as described previously (Yao et al., 2012b, 2013).

Cell-attached recordings were performed using an EPC-7 plus patchclamp amplifier (HEKA-Elektronic), and changes of membrane capacitance and conductance were measured as described previously (Yao et al., 2012b, 2013). The number of endocytic events per patch was counted as the total number of downward capacitance steps with sizes $>0.2 \mathrm{fF}$ within the first 5 min of recordings.

\section{Amperometry}

Conventional carbon fiber amperometry for catecholamine detection used $5-\mu \mathrm{m}$ carbon fibers (ALA Scientific Instruments, Farmingdale, NY) and was performed as described previously (Gong et al., 2005, 2007). The number of amperometrical spikes was counted as the total number of spikes with the amplitude $>5 \mathrm{pA}$ within $120 \mathrm{~s}$ after the stimulation initiation.
Competing interests

The authors declare no competing or financial interests.

\section{Author contributions}

K.T.V. and Z.J. performed and analyzed experiments on chromaffin cells under the supervision of L.W.G. F.B.Y. did the pulldown to identify dynamin. R.L.M. and V.B. performed all of the biochemical experiments and R.L.M. generated and designed the viral constructs under supervision of P.S.M. and W.S.S. R.L.M., L.W.G., P.S.M. and W.S.S. wrote the paper.

\section{Funding}

This work was supported by a Natural Sciences and Engineering Research Council of Canada (NESRC) grant to W.S.S.; a Canadian Institutes of Health Research [grant number MOP-15396 to P.S.M.]; and a National Science Foundation (NSF) award [grant number 1145581 to L.-W.G.]. W.S.S. and P.S.M. are James McGill Professors.

\section{References}

Artalejo, C. R., Lemmon, M. A., Schlessinger, J. and Palfrey, H. C. (1997) Specific role for the $\mathrm{PH}$ domain of dynamin-1 in the regulation of rapid endocytosis in adrenal chromaffin cells. EMBO J. 16, 1565-1574.

Barg, S. and Machado, J. D. (2008). Compensatory endocytosis in chromaffin cells. Acta Physiol. 192, 195-201.

Cardenas, A. M. and Marengo, F. D. (2010). Rapid endocytosis and vesicle recycling in neuroendocrine cells. Cell. Mol. Neurobiol. 30, 1365-1370.

de Heuvel, E., Bell, A. W., Ramjaun, A. R., Wong, K., Sossin, W. S. and McPherson, P. S. (1997). Identification of the major synaptojanin-binding proteins in brain. J. Biol. Chem. 272, 8710-8716.

Diril, M. K., Wienisch, M., Jung, N., Klingauf, J. and Haucke, V. (2006). Stonin 2 is an AP-2-dependent endocytic sorting adaptor for synaptotagmin internalization and recycling. Dev. Cell 10, 233-244.

Elvira, G., Wasiak, S., Blandford, V., Tong, X.-K., Serrano, A., Fan, X., del Rayo Sanchez-Carbente, M., Servant, F., Bell, A. W., Boismenu, D. et al. (2006). Characterization of an RNA granule from developing brain. Mol. Cell. Proteomics 5, 635-651.

Gong, L.-W., Di Paolo, G., Diaz, E., Cestra, G., Diaz, M.-E., Lindau, M., De Camilli, P. and Toomre, D. (2005). Phosphatidylinositol phosphate kinase type gamma regulates dynamics of large dense-core vesicle fusion. Proc. Natl. Acad. Sci. USA 102, 5204-5209.

Gong, L.-W., de Toledo, G. A. and Lindau, M. (2007). Exocytotic catecholamine release is not associated with cation flux through channels in the vesicle membrane but $\mathrm{Na}+$ influx through the fusion pore. Nat. Cell Biol. 9 915-922.

Gundelfinger, E. D., Kessels, M. M. and Qualmann, B. (2003). Temporal and spatial coordination of exocytosis and endocytosis. Nat. Rev. Mol. Cell Biol. 4 127-139.

Hilfiker, S., Pieribone, V. A., Nordstedt, C., Greengard, P. and Czernik, A. J. (1999). Regulation of synaptotagmin I phosphorylation by multiple protein kinases. J. Neurochem. 73, 921-932.

Huttner, W. B., Schiebler, W., Greengard, P. and De Camilli, P. (1983). Synapsin (protein I), a nerve terminal-specific phosphoprotein. III. Its association with synaptic vesicles studied in a highly purified synaptic vesicle preparation. J. Cell Biol. 96, 1374-1388.

Jung, N., Wienisch, M., Gu, M., Rand, J. B., Muller, S. L., Krause, G., Jorgensen, E. M., Klingauf, J. and Haucke, V. (2007). Molecular basis of synaptic vesicle cargo recognition by the endocytic sorting adaptor stonin 2. J. Cell Biol. 179, 1497-1510.

Lai, Y., Lou, X., Jho, Y., Yoon, T.-Y. and Shin, Y.-K. (2013). The synaptotagmin 1 linker may function as an electrostatic zipper that opens for docking but closes for fusion pore opening. Biochem. J. 456, 25-33.

Lv, J., Yang, Y., Yin, H., Chu, F., Wang, H., Zhang, W., Zhang, Y. and Jin, Y. (2009). Molecular determinants and evolutionary dynamics of wobble splicing. Mol. Biol. Evol. 26, 1081-1092.

Marqueze, B., Boudier, J. A., Mizuta, M., Inagaki, N., Seino, S. and Seagar, M. (1995). Cellular localization of synaptotagmin I, II, and III mRNAs in the central nervous system and pituitary and adrenal glands of the rat. J. Neurosci. 15, 4906-4917.

Nagy, G., Kim, J. H., Pang, Z. P., Matti, U., Rettig, J., Sudhof, T. C. and Sorensen, J. B. (2006). Different effects on fast exocytosis induced by synaptotagmin 1 and 2 isoforms and abundance but not by phosphorylation. J. Neurosci. 26 632-643.

Nakhost, A., Houeland, G., Castellucci, V. F. and Sossin, W. S. (2003) Differential regulation of transmitter release by alternatively spliced forms of synaptotagmin I. J. Neurosci. 23, 6238-6244.

Nicholson-Tomishima, K. and Ryan, T. A. (2004). Kinetic efficiency of endocytosis at mammalian CNS synapses requires synaptotagmin I. Proc. Natl. Acad. Sci. USA 101, 16648-16652. 
Poskanzer, K. E., Marek, K. W., Sweeney, S. T. and Davis, G. W. (2003). Synaptotagmin I is necessary for compensatory synaptic vesicle endocytosis in vivo. Nature 426, 559-563.

Ramachandran, R., Pucadyil, T. J., Liu, Y.-W., Acharya, S., Leonard, M., Lukiyanchuk, V. and Schmid, S. L. (2009). Membrane insertion of the pleckstrin homology domain variable loop 1 is critical for dynamin-catalyzed vesicle scission. Mol. Biol. Cell 20, 4630-4639.

Ritter, B., Murphy, S., Dokainish, H., Girard, M., Gudheti, M. V., Kozlov, G., Halin, M., Philie, J., Jorgensen, E. M., Gehring, K. et al. (2013). NECAP 1 regulates $\mathrm{AP}-2$ interactions to control vesicle size, number, and cargo during clathrin-mediated endocytosis. PLoS Biol. 11, e1001670.

Smith, S. M., Renden, R. and von Gersdorff, H. (2008). Synaptic vesicle endocytosis: fast and slow modes of membrane retrieval. Trends Neurosci. 31, 559-568.
Yao, J., Kwon, S. E., Gaffaney, J. D., Dunning, F. M. and Chapman, E. R. (2012a). Uncoupling the roles of synaptotagmin I during endo- and exocytosis of synaptic vesicles. Nat. Neurosci. 15, 243-249.

Yao, L.-H., Rao, Y., Varga, K., Wang, C.-Y., Xiao, P., Lindau, M. and Gong, L.-W. (2012b). Synaptotagmin 1 is necessary for the Ca2+ dependence of clathrinmediated endocytosis. J. Neurosci. 32, 3778-3785.

Yao, L.-H., Rao, Y., Bang, C., Kurilova, S., Varga, K., Wang, C.-Y., Weller, B. D., Cho, W., Cheng, J. and Gong, L.-W. (2013). Actin polymerization does not provide direct mechanical forces for vesicle fission during clathrin-mediated endocytosis. J. Neurosci. 33, 15793-15798.

Zhang, J. Z., Davletov, B. A., Südhof, T. C. and Anderson, R. G. W. (1994) Synaptotagmin $\mathrm{I}$ is a high affinity receptor for clathrin AP-2: implications for membrane recycling. Cell 78, 751-760. 